\title{
ERRATUM
}

Keiko Yamazaki · Masakazu Takazoe · Torao Tanaka

Toshiki Ichimori · Yusuke Nakamura

\section{Absence of mutation in the NOD2/CARD15 gene among 483 Japanese patients with Crohn's disease}

Published online: 17 June 2003

(C) The Japan Society of Human Genetics and Springer-Verlag 2003

\section{J Hum Genet (2002) 47:469-472}

The name of one author, Toshiki Kazumori, should be corrected to Toshiki Ichimori.

K. Yamazaki · Y. Nakamura $(\bowtie)$

Laboratory of Molecular Medicine, Institute of Medical Science,

University of Tokyo, 4-6-1 Shirokanedai, Minato-ku,

Tokyo 108-8639, Japan

E-mail: yusuke@ims.u-tokyo.ac.jp

Tel.: + 81-3-5449-5372

Fax: +81-3-5449-5433

M. Takazoe $\cdot$ T. Tanaka $\cdot$ T. Ichimori

Department of Medicine, Division of Gastroenterology,

Social Insurance Chuo General Hospital, Tokyo, Japan 